\title{
Economic Evaluation of Anticyclic Citrullinated Peptide Positivity in Rheumatoid Arthritis
}

\author{
JaeJin An, BPharm, PhD; Zoe Bider-Canfield, MPH; Jenny Kang, PharmD; Evo Alemao, RPh, PhD; \\ Sean E. Connolly, PhD; Antony T. Lin, MD; and T. Craig Cheetham, PharmD, MS
}

\begin{abstract}
BACKGROUND: Anticyclic citrullinated peptide (anti-CCP) positivity may be a strong predictor of joint erosion and a potential biomarker for guiding treatment decisions for rheumatoid arthritis (RA). However, limited studies are currently available on the effect of anti-CCP positivity on health care utilization and/or medical costs of RA patients.
\end{abstract}

OBJECTIVE: To investigate short-term and long-term direct health care expenditures associated with anti-CCP positivity in newly diagnosed RA patients.

METHODS: A retrospective cohort study was conducted in adult RA patients within a U.S. integrated health care delivery system (January 1, 2007-June 30, 2015). Patients were required to have 2 RA diagnoses and treatment with a conventional or biologic disease-modifying antirheumatic drug (DMARD) within 12 months. The first RA diagnosis date was labeled as the index date, and patients were followed until they left the health plan, died, or reached the end of the study period. Patient demographics, anti-CCP results, comorbid conditions, and health care resource utilization during baseline (12 months before the index date) and follow-up periods were collected. Nationally recognized direct medical costs were assigned to health care utilization to calculate health care costs in 2015 U.S. dollars. The baseline differences between anti-CCP positivity and negativity and differences in censoring during follow-up were addressed using propensity scores. The mean differences in costs were estimated using recycled prediction methods.

RESULTS: 2,448 newly diagnosed RA patients were identified and followed for a median of 3.7 years (range $=1-8$ years). At baseline, $65.8 \%$ of patients were anti-CCP positive. Anti-CCP-positive patients had fewer comorbid conditions at baseline. During the first 12 months of follow-up, median (interquartile range) total health care expenditures for anti-CCP-positive and anti-CCP-negative patients were $\$ 6,200(\$ 3,563-\$ 13,260)$ and $\$ 7,022$ $(\$ 3,885-\$ 12,995)$, respectively. After adjusting for baseline differences, total incremental mean cost associated with anti-CCP positivity during the first 12 months was estimated to be $\$ 2,163$ per patient $(P=0.001)$. The annual incremental costs in anti-CCP-positive patients became progressively larger over time, from $\$ 2,163$ during the first year to $\$ 5,062$ during the fourth year. Anti-CCP positivity was associated with higher prescription, laboratory testing, and rheumatologist utilization. A higher percentage of anti-CCP-positive patients received 1 or more biologic DMARDs (11.6\% for anti-CCP-positive vs. $5.7 \%$ for anti-CCP negative; $P<0.001$ ) compared with anti-CCP-negative patients during the 12-month follow-up, which resulted in $\$ 2,499$ in incremental prescription costs $(P<0.001)$. Total additional burden associated with anti-CCP positivity during the first 4 years was estimated to be $\$ 14,089$ per patient.

CONCLUSIONS: In newly diagnosed RA patients, higher economic burden associated with anti-CCP positivity was mainly driven by prescription costs.

J Manag Care Spec Pharm. 2019;25(4):469-77

Copyright $\odot 2019$, Academy of Managed Care Pharmacy. All rights reserved.

\section{What is already known about this subject}

The presence of a positive anticyclic citrullinated peptide (antiCCP) test has been associated with a more destructive course of disease, disease severity, radiographic erosions, disability, and mortality in rheumatoid arthritis (RA)

A recent study suggested higher economic burden in anti-CCPpositive patients during the first 12 months after RA diagnosis.

\section{What this study adds}

This study estimated the long-term economic impact of anti-CCP positivity in patients with RA

Total additional burden associated with anti-CCP positivity during the first 4 years from RA diagnosis was estimated to be $\$ 14,089$

The higher economic burden associated with anti-CCP positivity was primarily due to prescription costs, and this burden persisted across all years of follow-up.

$\mathrm{R}$ heumatoid arthritis (RA) is one of the most common autoimmune diseases, affecting nearly 1.3 million people in the United States. ${ }^{1}$ RA is characterized by chronic inflammation of the joints, which can ultimately lead to cartilage and bone destruction. ${ }^{2}$ RA severely affects the quality of life for patients and has major economic consequences for society. $^{3}$ One U.S. study estimated that total annual RA costs for privately insured and Medicare populations were $\$ 306$ million and $\$ 600$ million (in 2005 U.S. dollars), respectively. ${ }^{4}$

Early identification of RA before any joint erosion is an important step toward optimizing long-term prognosis. Serologic markers are recommended to diagnose RA patients early and to further identify patients who may benefit from early aggressive treatment., ${ }^{5,6}$ Anticyclic citrullinated peptide (anti-CCP) antibodies are one of the serologic markers recommended by the American College of Rheumatology and the European League Against Rheumatism; they have become popular due to their improved specificity and sensitivity in RA compared with rheumatoid factor testing. ${ }^{7-10}$ Our previous findings revealed that the majority of RA patients in an integrated health care delivery system received anti-CCP testing at the time of RA diagnosis. ${ }^{11}$

The presence of a positive anti-CCP test has been associated with a more destructive course of disease, disease severity, 
radiographic erosions, disability, and mortality. ${ }^{12-15}$ Mjaavatten et al. (2010) reported that increasing levels of rheumatoid factor and anti-CCP were associated with persistent joint inflammation. ${ }^{16}$ In addition, a recent systematic literature review analyzing 25 studies found that anti-CCP levels are strong predictors of severity in RA. ${ }^{17}$ Treatment strategies could be different for these anti-CCP-positive patients; however, further clinical studies are needed to support specific treatment regimens for this group of patients. ${ }^{18}$

A better understanding of health care utilization patterns and total health care expenditures for anti-CCP-positive patients will help to support the need for targeted therapies. Limited studies are currently available on the effect of anti-CCP positivity on the health care utilization and/or medical costs of RA patients. ${ }^{19,20}$ While currently available data seem to suggest a higher economic burden in anti-CCP-positive patients during the first 12 months after RA diagnosis, economic data beyond the first year are not yet available. ${ }^{20}$ Therefore, this study aimed to (a) describe and compare health care resource utilization based on anti-CCP sero-status at the time of RA diagnosis and (b) determine the association between anti-CCP positivity and short-term and long-term direct medical care costs among patients newly diagnosed with RA in an integrated health care delivery system.

\section{Methods}

\section{Study Setting}

Kaiser Permanente Southern California (KPSC) is a nonprofit, integrated health care delivery system with a membership of over 4.0 million. The population served by Kaiser Permanente is socioeconomically diverse and broadly representative of the racial/ethnic groups living in Southern California and across the United States. ${ }^{21}$ KPSC provides integrated, comprehensive medical services through its own facilities, which include 16 hospitals and more than 200 outpatient clinics. Each KPSC member receives a unique medical record number that they keep for life and that allows linkage to various clinical and administrative databases, including member enrollment and benefits, hospital discharge diagnoses and procedures, visits to physician's offices (diagnosis and procedures), radiology, laboratory test results, and drug dispensing. All aspects of care are captured in a continuously updated, comprehensive electronic medical record (EMR) that is available for research purposes.

\section{Study Design}

This retrospective cohort study, conducted within KPSC, investigated anti-CCP test results (sero-positivity) and their associations with health care resource utilization and costs. Patients with a new RA diagnosis from January 1, 2007, to June 30, 2014, were identified and followed until they left the health plan, died, or reached the end of the study period (June 30, 2015), whichever occurred first. The study was approved by the KPSC Institutional Review Board; the requirement for written informed consent was waived due to the observational nature of the study.

\section{Study Cohort}

Patients were required to have 2 RA diagnoses (International Classification of Diseases, Ninth Revision, Clinical Modification [ICD-9-CM] code 714.x) from January 1, 2007, to June 30, 2014, and treatment with a conventional or biologic disease-modifying antirheumatic drug (DMARD) within 12 months of the first RA diagnosis. ${ }^{22}$ The DMARD prescription was identified using Generic Product Identifier codes (Appendix A, available in online article). The first RA diagnosis date was labeled as the index date; eligible patients were required to have 12 months of continuous membership plus drug benefit coverage before their index date (baseline period). Patients were also required to have 12 months of post-index continuous membership to determine short-term health care utilization and costs following their RA diagnosis. The pre-index period was used to determine a patient's baseline characteristics and to identify whether the patient was a newly diagnosed (incident) RA case. Patients with RA diagnoses or DMARD therapy during the pre-index period were excluded from the study to make sure we included only incident RA cases. Patients with ICD-9-CM codes of 714.3 (juvenile arthritis) or 714.9 (polyrheumatic arthropathy) or autoimmune conditions other than RA were also excluded from the study (Appendix A lists autoimmune conditions and ICD-9-CM codes). Patients without any anti-CCP testing were also excluded, since the purpose of this study was to compare outcomes based on anti-CCP test sero-positivity.

\section{Anti-CCP Testing}

Baseline anti-CCP test results included any anti-CCP tests before the second confirmatory RA diagnosis or the first DMARD prescription dispense date to evaluate anti-CCP testing at the time of confirmatory diagnosis or at treatment with DMARDs for the incident cohort. In cases of multiple testing, the test closest to the first RA diagnosis was defined as the baseline anti-CCP test. Based on the test results, incident RA patients were classified into 2 sero-positive patient groups: anti-CCP positive and anti-CCP negative. The determination of test positivity and negativity followed the laboratory testing specifications conducted during the study time period (Table 1).

\section{Study Outcomes}

Health Care Utilization. From the EMR, every health care encounter was collected and summarized by the type of service the patient received. We classified health care utilization by outpatient visits including urgent care, laboratory testing, prescriptions, emergency department (ED) visits, and inpatient hospitalizations. We also collected diagnosis or procedure codes and physician specialty associated with each visit. The number 
TABLE 1 Baseline Characteristics by Anti-CCP Test Results ${ }^{a}$

\begin{tabular}{|c|c|c|c|c|c|c|c|}
\hline \multirow[b]{2}{*}{ Mean age (SD) } & \multicolumn{2}{|c|}{$\begin{array}{c}\text { Anti-CCP Positive } \\
(\mathrm{n}=1,612)\end{array}$} & \multicolumn{2}{|c|}{$\begin{array}{l}\text { Anti-CCP Negative } \\
(\mathrm{n}=836)\end{array}$} & \multicolumn{2}{|c|}{$\begin{array}{c}\text { Total } \\
(\mathrm{N}=2,448)\end{array}$} & \multirow{2}{*}{$\begin{array}{r}P \text { Value } \\
0.0003\end{array}$} \\
\hline & 54.7 & $(14.2)$ & 56.9 & $(14.4)$ & 55.5 & $(14.3)$ & \\
\hline Female, n (\%) & 1,199 & $(74.4)$ & 655 & (78.3) & 1,854 & $(75.7)$ & 0.0298 \\
\hline Race/ethnicity, n (\%) & & & & & & & $<0.0001$ \\
\hline Asian & 129 & $(8.0)$ & 71 & $(8.5)$ & 200 & $(8.2)$ & \\
\hline Black & 223 & (13.8) & 66 & $(7.9)$ & 289 & $(11.8)$ & \\
\hline Hispanic & 689 & $(42.7)$ & 306 & $(36.6)$ & 995 & $(40.6)$ & \\
\hline White & 522 & $(32.4)$ & 365 & $(43.7)$ & 887 & $(36.2)$ & \\
\hline Others/unknown & 49 & $(3.0)$ & 28 & (3.3) & 77 & (3.1) & \\
\hline Smoking status, n (\%) & & & & & & & 0.0006 \\
\hline Current & 206 & (12.8) & 61 & (7.3) & 267 & (10.9) & \\
\hline Former & 367 & $(22.8)$ & 204 & $(24.4)$ & 571 & $(23.3)$ & \\
\hline Never & 939 & $(58.3)$ & 520 & $(62.2)$ & 1,459 & $(59.6)$ & \\
\hline Unknown & 100 & $(6.2)$ & 51 & $(6.1)$ & 151 & $(6.2)$ & \\
\hline Elixhauser category, n (\%) & & & & & & & $<0.0001$ \\
\hline $0-1$ & 235 & $(14.6)$ & 84 & $(10.0)$ & 319 & $(13.0)$ & \\
\hline $2-3$ & 609 & $(37.8)$ & 267 & $(31.9)$ & 876 & $(35.8)$ & \\
\hline $4-5$ & 422 & $(26.2)$ & 258 & $(30.9)$ & 680 & $(27.8)$ & \\
\hline $6-15$ & 346 & $(21.5)$ & 227 & $(27.2)$ & 573 & $(23.4)$ & \\
\hline BMI category, n (\%) & & & & & & & 0.1363 \\
\hline$<25$ & 541 & (33.6) & 281 & (33.6) & 822 & (33.6) & \\
\hline $25-29$ & 476 & $(29.5)$ & 218 & $(26.1)$ & 694 & $(28.3)$ & \\
\hline$\geq 30$ & 595 & (36.9) & 337 & $(40.3)$ & 932 & $(38.1)$ & \\
\hline Steroid use, n (\%) & 1,060 & $(65.8)$ & 525 & $(62.8)$ & 1,585 & $(64.7)$ & 0.1463 \\
\hline NSAID use, n (\%) & 1,265 & $(78.5)$ & 609 & $(72.8)$ & 1,874 & $(76.6)$ & 0.0018 \\
\hline First DMARDs ${ }^{\mathrm{b}}, \mathrm{n}(\%)$ & & & & & & & 0.8197 \\
\hline Biologic DMARDs & 17 & $(1.1)$ & 8 & $(1.0)$ & 25 & $(1.0)$ & \\
\hline Conventional DMARDs & 1,595 & (98.9) & 828 & $(99.0)$ & 2,423 & $(99.0)$ & \\
\hline Baseline CRP or ESR, n (\%) & & & & & & & $<0.0001$ \\
\hline CRP or ESR positive & 1,135 & $(70.4)$ & 427 & $(51.1)$ & 1,562 & $(63.8)$ & \\
\hline Not CRP/ESR positive & 444 & $(27.5)$ & 373 & $(44.6)$ & 817 & $(33.4)$ & \\
\hline Both CRP/ESR missing & 33 & $(2.0)$ & 36 & $(4.3)$ & 69 & $(2.8)$ & \\
\hline Mean (SD) CIRAS scores & 7.0 & $(1.3)$ & 6.9 & $(1.3)$ & 7.0 & $(1.3)$ & 0.0604 \\
\hline Mean (SD) follow-up, in days & $1,428.6$ & $(773.4)$ & $1,511.4$ & $(772.9)$ & $1,456.9$ & $(774.1)$ & 0.0077 \\
\hline Median (IQR) follow-up, in days & $1,317.5$ & $35.0-2,045.0)$ & 1,406 & $26.0-2,196.0)$ & $1,348.5$ & $66.0-2,092.5)$ & 0.0077 \\
\hline
\end{tabular}

${ }^{a}$ CCP IGG test using the chemiluminescent microparticle immunoassay before July 2010 (reference range <5.0 U/mL); CCP antibody using the enzyme-linked immunosorbent immunoassay (reference range <20 units) before July 2010.

${ }^{b}$ First DMARDs are at the index date or within 12 months from the index date.

$B M I=$ body mass index; $C C P=$ cyclic citrullinated peptide; $C I R A S=$ claims-based index for $R A$ severity; $C R P=C$-reactive protein; DMARD = disease-modifying antirheumatic drug; ESR=erythrocyte sedimentation rate; IGG=immunoglobulin $G$; IQR=interquartile range; NSAID = nonsteroidal anti-inflammatory drug; SD=standard deviation.

of rheumatologist visits and biologic DMARD use were summarized separately as RA-specific health care resource utilization.

Direct Health Care Expenditures. To estimate costs, we assigned nationally recognized direct health care costs to each health care visit. ICD-9-CM and Current Procedural Terminology (CPT) codes associated with each outpatient visit, ED visit, and laboratory test were mapped to the Centers for Medicare \& Medicaid Services' Physician Fee Schedule for these costs. ${ }^{23}$ National Inpatient Sample 2013 data were used to estimate the inpatient cost based on a patient's diagnosisrelated group, length of stay, and use of major operating room during the stay. ${ }^{24}$ The average wholesale price from RED BOOK was used to estimate prescription costs..$^{25}$ All costs were calculated in 2015 U.S. dollars, applying the Consumer Price Index for medical care services. ${ }^{26}$

\section{Statistical Analysis}

We compared the 2 RA groups, those with positive anti-CCP and those with negative anti-CCP test results at baseline. Descriptive statistics were used to compare baseline differences including the claims-based index for RA severity (CIRAS) scores between anti-CCP positivity and negativity and health care utilization by anti-CCP category. The CIRAS score was developed to capture the severity of RA using health care 
TABLE 2 Health Care Utilization by Anti-CCP Category

\begin{tabular}{|c|c|c|c|c|}
\hline Type of Encounter & $\begin{array}{l}\text { Anti-CCP Positive } \\
(\mathrm{n}=1,612)\end{array}$ & $\begin{array}{l}\text { Anti-CCP Negative } \\
\quad(\mathbf{n}=836)\end{array}$ & $\begin{array}{c}\text { Total } \\
(\mathrm{N}=2,448)\end{array}$ & $P$ Value \\
\hline \multicolumn{5}{|c|}{ Outpatient office visits, n (\%) } \\
\hline Baseline & $1,548 \quad(96.0)$ & $817 \quad(97.7)$ & $2,365 \quad(96.6)$ & 0.0334 \\
\hline Follow-up (1 year) & $1,592(98.8)$ & $832(99.5)$ & $2,424 \quad(99.0)$ & 0.0835 \\
\hline Follow-up (alla) & $1,606 \quad(99.6)$ & $836 \quad(100.0)$ & $2,442 \quad(99.8)$ & 0.1011 \\
\hline \multicolumn{5}{|c|}{ Office visits, median (IQR) } \\
\hline Baseline & $11(6.0-21.0)$ & $15.5(9.0-26.0)$ & $13(7.0-23.0)$ & $<0.0001$ \\
\hline Follow-up (1 year) & $16(10.0-26.0)$ & $19(12.0-31.0)$ & $17(11.0-27.0)$ & $<0.0001$ \\
\hline Follow-up (alla) & $52(27.0-96.0)$ & $68 \quad(32.5-128.0)$ & $56.5(29.0-104.0)$ & $<0.0001$ \\
\hline \multicolumn{5}{|c|}{ Emergency visits, n (\%) } \\
\hline Baseline & $218 \quad(13.5)$ & $112(13.4)$ & $330(13.5)$ & 0.9308 \\
\hline Follow-up (1 year) & $224(13.9)$ & $113(13.5)$ & $337(13.8)$ & 0.7963 \\
\hline Follow-up (alla) & $582(36.1)$ & $331 \quad(39.6)$ & $913 \quad(37.3)$ & 0.0905 \\
\hline \multicolumn{5}{|l|}{ Hospitalizations, n (\%) } \\
\hline Baseline & $167(10.4)$ & $101 \quad(12.1)$ & $268(10.9)$ & 0.1958 \\
\hline Follow-up (1 year) & $179(11.1)$ & $108(12.9)$ & $287(11.7)$ & 0.1857 \\
\hline Follow-up (alla) & $463(28.7)$ & $297(35.5)$ & $760 \quad(31.0)$ & 0.0006 \\
\hline \multicolumn{5}{|c|}{ Biologic DMARD use, $\mathrm{n}(\%)$} \\
\hline Baseline & - & - & - & - \\
\hline Follow-up (1 year) & $187(11.6)$ & $48(5.7)$ & $235(9.6)$ & $<0.0001$ \\
\hline Follow-up (alla) & $361 \quad(22.4)$ & $106(12.7)$ & 467 (19.1) & $<0.0001$ \\
\hline \multicolumn{5}{|c|}{ Rheumatologist visits, median (IQR) } \\
\hline Baseline & $0(0-1)$ & $1(0-1)$ & $0 \quad(0-1)$ & $<0.0001$ \\
\hline Follow-up (1 year) & $6(4-8)$ & $4(3-7)$ & $5(3-8)$ & $<0.0001$ \\
\hline Follow-up (alla) & $13(7-22)$ & $9 \quad(5-17.5)$ & $11 \quad(6-21)$ & $<0.0001$ \\
\hline
\end{tabular}

utilization data such as the number of inflammatory marker tests ordered and the number of platelet counts ordered. ${ }^{27} \mathrm{Chi}-$ square tests were used for categorical variables, while t-tests or Wilcoxon signed-rank tests were used for continuous variables.

For the initial 12-month follow-up, a difference-in-difference (DID) propensity score-adjusted analysis was conducted to estimate the difference in health care costs from the baseline period to the 12-month follow-up period in anti-CCP-positive and anti-CCP-negative groups. ${ }^{28}$ DID is a statistical technique used to control for background changes in outcomes that occur over time. ${ }^{29}$ We further applied the propensity score methods to control for baseline differences between anti-CCP-positive and anti-CCP-negative patients. A generalized linear regression model (gamma distribution, log link) with recycled prediction methods was used to quantify mean differences in costs. ${ }^{30}$

Long-term health care costs were collected during the entire 8 -year follow-up period. Due to the presence of censored patient follow-up data, we used an approach to estimate population mean costs, accounting for censoring in the data using inverse probability weighting to investigate long-term differences in costs between anti-CCP-positive and anti-CCP-negative groups. ${ }^{31-33}$ To do this, we partitioned each patient's followup into 1-year periods of time, and weights were calculated for missing values using the inverse of probability of not being censored given race, age, gender, and Elixhauser Comorbidity Index score in each interval. ${ }^{34} \mathrm{~A}$ logistic regression was used to estimate the inverse-probability-of-censoring-weights (IPW).

Partitioned IPW least squares regression analyses were performed to compare adjusted cumulative costs over 8 years of follow-up time between anti-CCP-positive and anti-CCPnegative groups. A separate generalized linear regression model (gamma distribution, log link) among patients who completed follow-up during each time interval applying IPW was conducted. Recycled prediction methods were used to predict the mean cost in the anti-CCP-positive and anti-CCP-negative groups for each time interval. The predicted mean cost within each partition was summed across partitions to estimate the cumulative costs in the cohort while accounting for censoring All statistical analyses were performed with SAS version 9.3 for Windows (SAS Institute, Cary, NC). A $P$ value $<0.05$ was considered statically significant.

\section{Results}

A total of 2,448 newly diagnosed RA patients were identified with a mean (standard deviation [SD]) age of 55.5 (14.3) years and $75.7 \%$ being female. The majority of patients were 


\section{FIGURE 1 Expected Mean Differences in Incremental Health Care Expenditure per Patient Before and} 1 Year After RA Diagnosis ${ }^{\mathrm{a}}$

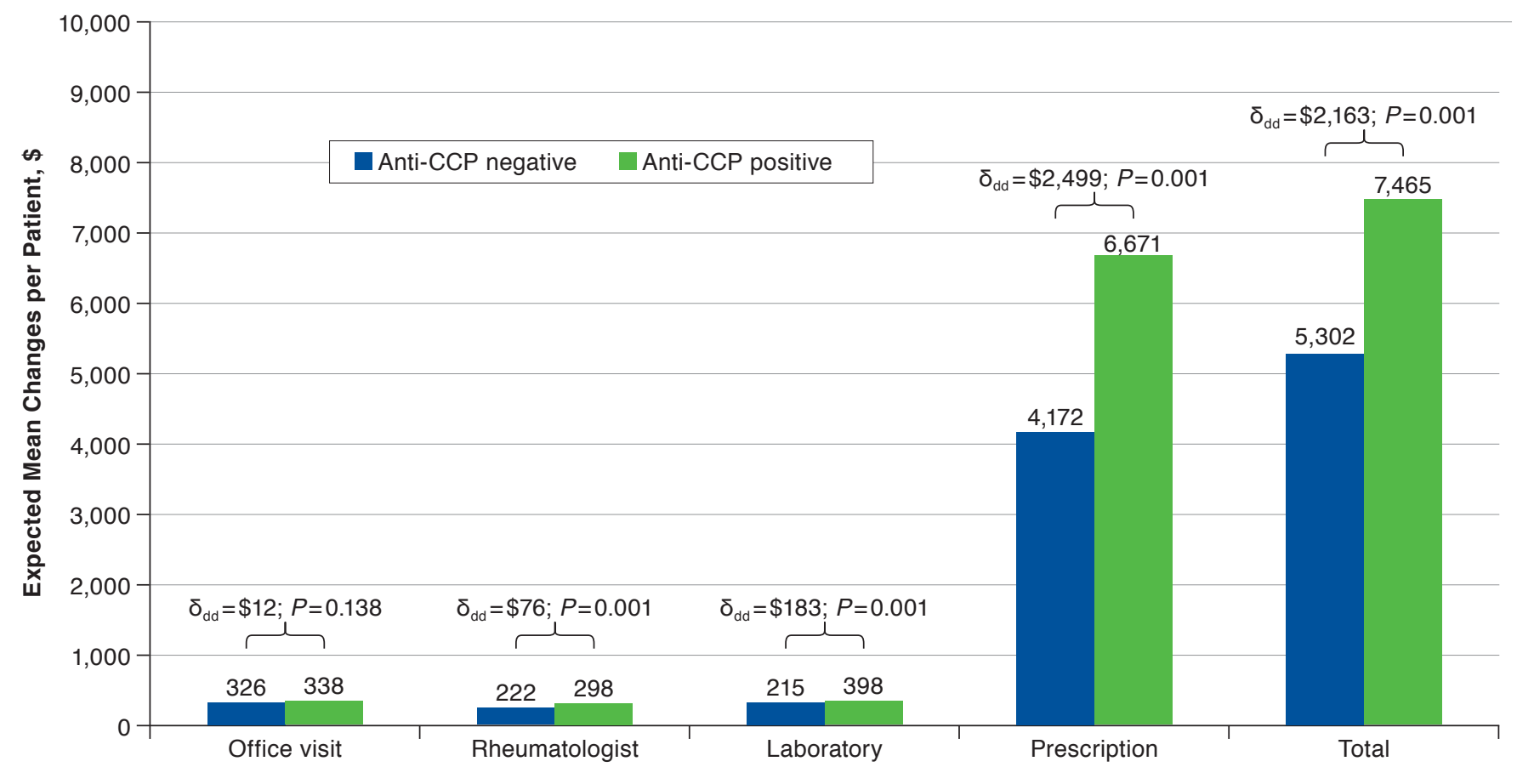

Note: Estimated mean changes were calculated using recycled prediction methods after the generalized linear models using gamma distribution, log link. $P$ values were

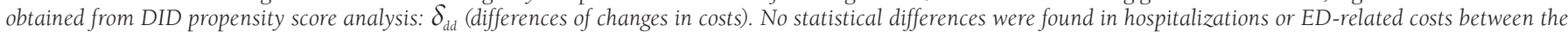
2 groups; therefore, this was not shown in this graph.

a Differences in costs are shown in 2015 U.S. dollars.

$C C P=$ cyclic citrullinated peptide $; D I D=$ difference-in-difference $; E D=$ emergency department RA =rheumatoid arthritis .

Hispanic (40.6\%), followed by white (36.2\%) and black (11.8\%). The median follow-up was 3.7 years (interquartile range $[\mathrm{IQR}]=2.1-5.7$ years). A total of 1,117 patients had 4 years of follow-up, and 83 patients completed 8 years of follow-up.

At baseline, 1,612 (65.8\%) patients had an anti-CCP-positive test result, and 836 (34.2\%) had an anti-CCP-negative test result. Baseline characteristics of the anti-CCP-positive and anti-CCP-negative patients are shown in Table 1. A higher percentage of Hispanics, blacks, and current smokers were found in the anti-CCP-positive group compared with the antiCCP-negative group $(P<0.05)$. In addition, a higher percentage of anti-CCP-positive patients were on nonsteroidal anti-inflammatory drugs and had elevated C-reactive protein or erythrocyte sedimentation rate compared with anti-CCP-negative patients $(P<0.05)$. In contrast, fewer comorbidities were found among anti-CCP-positive patients compared with anti-CCPnegative patients (Elixhauser comorbidity score of $\geq 6: 21.5 \%$ for anti-CCP-positive vs. 27.2\% for anti-CCP-negative patients; $P<0.001)$.

\section{Health Care Utilization}

Baseline and follow-up health care utilization by anti-CCP test results is shown in Table 2. Almost all RA patients had 1 or more office visits at baseline and during follow-up. Patients with anti-CCP-positive test results had a lower number of office visits at baseline compared with patients with antiCCP-negative results (median office visits $=11 \quad[\mathrm{IQR}=6-21]$ for anti-CCP-positive vs. 15.5 [IQR=9-26] for anti-CCPnegative patients; $P<0.001)$. A lower number of office visits for the anti-CCP-positive group was also found during the first 12 months after RA diagnosis (median office visits = 16 $[\mathrm{IQR}=10-26]$ for anti-CCP-positive vs. 19 [IQR=12-31] for anti-CCP-negative patients; $P<0.001$ ). However, anti-CCP-positive patients had more frequent rheumatologist visits (median rheumatologist visits $=6[\mathrm{IQR}=4-8]$ for anti-CCP-positive vs . 4 [IQR = 3-7] for anti-CCP-negative patients; $P<0.001$ ), and a higher percentage of anti-CCP-positive patients received 1 or more biologic DMARDs (11.6\% for anti-CCP-positive vs. $5.7 \%$ for anti-CCP-negative patients; $P<0.001$ ) compared with anti-CCP-negative patients during the 12-month follow-up. 
TABLE 3 12-Month Direct Health Care Cost Differences by Anti-CCP Category ${ }^{\mathrm{a}}$

\begin{tabular}{|c|c|c|c|c|c|c|c|}
\hline Type of Encounter & Label & Beta Estimate & SE & $P$ Value & $\begin{array}{c}\text { Estimated Mean } \\
\text { in Anti-CCP- } \\
\text { Positive } \\
\text { Patients, \$ }\end{array}$ & $\begin{array}{c}\text { Estimated Mean } \\
\text { in Anti-CCP- } \\
\text { Negative } \\
\text { Patients, \$ }\end{array}$ & $\begin{array}{l}\text { Estimated Mean } \\
\text { Differences in } \\
\text { Anti-CCP-Positive } \\
\text { and Anti-CPP- } \\
\text { Negative Patients }{ }^{\mathrm{b}}, \$\end{array}$ \\
\hline \multirow[t]{2}{*}{ Office visit } & Unadjusted & 0.05 & 0.04 & 0.176 & & & \\
\hline & PS weighted & 0.06 & 0.04 & 0.138 & 338 & 326 & 12 \\
\hline \multirow[t]{2}{*}{ Rheumatologist visit } & Unadjusted & 0.26 & 0.07 & 0.0001 & & & \\
\hline & PS weighted & 0.29 & 0.07 & $<0.0001$ & 298 & 222 & $76^{c}$ \\
\hline \multirow[t]{2}{*}{ Laboratory testing } & Unadjusted & 0.22 & 0.04 & $<0.0001$ & & & \\
\hline & PS weighted & 0.23 & 0.04 & $<0.0001$ & 398 & 215 & $183^{c}$ \\
\hline \multirow[t]{2}{*}{ Prescription } & Unadjusted & 0.43 & 0.07 & $<0.0001$ & & & \\
\hline & PS weighted & 0.46 & 0.08 & $<0.0001$ & 6,671 & 4,172 & $2,499^{c}$ \\
\hline \multirow[t]{2}{*}{$\overline{\mathrm{ED}}$} & Unadjusted & -0.11 & 0.21 & 0.607 & & & \\
\hline & PS weighted & 0.06 & 0.23 & 0.809 & 1 & -3 & 3 \\
\hline \multirow[t]{2}{*}{ Hospitalization } & Unadjusted & -0.20 & 0.28 & 0.467 & & & \\
\hline & PS weighted & -0.26 & 0.31 & 0.406 & 52 & 654 & -602 \\
\hline \multirow[t]{2}{*}{ Total } & Unadjusted & 0.24 & 0.08 & 0.003 & & & \\
\hline & PS weighted & 0.25 & 0.08 & 0.001 & 7,465 & 5,302 & $2,163^{c}$ \\
\hline
\end{tabular}

Note: These results are from the generalized linear models using difference-in-difference unadjusted and propensity score methods. Variables used in the propensity score models and regression models were age, gender, racelethnicity, and Elixhauser Comorbidity Index score.

${ }^{a}$ Cost differences are shown in 2015 U.S. dollars.

${ }^{b}$ Estimated mean changes were calculated using recycled prediction methods after the generalized linear models using gamma distribution, log link.

cStatistically significant findings $(P<0.05)$.

$C C P=$ cyclic citrullinated peptide; $E D=$ emergency department; $P S=$ propensity score; $S E=$ standard error

No statistical differences were found for ED visits or hospitalizations between anti-CCP-positive and anti-CCP-negative patients. Long-term health care utilization also showed that anti-CCP-positive patients had a higher number of rheumatologist visits (median rheumatologist visits: 13 [IQR $=7-22]$ for anti-CCP-positive vs. 9 [IQR =5-17.5] for anti-CCPnegative patients; $P<0.001$ ) and were likely to receive 1 or more biologic DMARDs (22.4\% for anti-CCP-positive vs. $12.7 \%$ for anti-CCP-negative patients; $P<0.001)$ compared with anti$\mathrm{CCP}$-negative patients. The percentage of patients with hospitalizations was higher for anti-CCP-negative patients for longterm follow-up compared with anti-CCP-positive patients.

\section{Health Care Expenditures}

During the first 12 months of follow-up, median (IQR) total health care expenditures for anti-CCP-positive and anti-CCPnegative patients were $\$ 6,200(\$ 3,563-\$ 13,260)$ and $\$ 7,022$ $(\$ 3,885-\$ 12,995)$, respectively (Appendix B, available in online article). The largest proportion of expenditures was prescription costs, followed by outpatient office visits and laboratory tests. The median (IQR) prescription expenditures for antiCCP-positive and anti-CCP-negative patients were $\$ 3,756$ $(\$ 1,798-\$ 8,373)$ and $\$ 4,417(\$ 2,072-\$ 8,268)$, respectively.

Using the DID propensity score approach and adjusting for baseline differences in costs, patient demographics, and comorbidities, anti-CCP positivity was associated with higher mean change in prescription $\left(\delta_{\mathrm{dd}}=\$ 2,499 ; \quad P<0.001\right)$, laboratory testing $\left(\delta_{\mathrm{dd}}=\$ 183 ; P<0.001\right)$, and rheumatologist visit costs $\left(\delta_{\mathrm{dd}}=\$ 76 ; P<0.001\right)$ during the first 12 months of follow-up (Table 3). Total 1-year incremental cost associated with antiCCP positivity was estimated to be $\$ 2,163(P=0.001)$. No statistical differences were found in hospitalizations or ED-related costs between the 2 groups (Figure 1).

Predicted cumulative total health care costs were estimated for anti-CCP-positive and anti-CCP-negative groups separately. At 4 years of follow-up, cumulative total direct medical costs were estimated to be $\$ 61,248$ per person for the anti-CCP-positive group, while the costs of the anti-CCP-negative group were estimated to be $\$ 47,159$ (Table 4). The total additional burden associated with anti-CCP positivity during the first 4 years since RA diagnosis was $\$ 14,089$. The annual incremental costs in anti-CCP-positive patients became progressively larger over time, from $\$ 2,163$ during the first year to $\$ 5,062$ during the fourth year. Most of the increased economic burden associated with anti-CCP positivity was again from prescription expenditures. Over the first 4 years, additional costs for anti-CCPpositive patients were estimated to be $\$ 15,806$ for prescriptions, $\$ 435$ for laboratory testing, and $\$ 390$ for rheumatologist visits, while anti-CCP positivity was associated with a decrease in $\$ 965$ for outpatient office visits compared with anti-CCP negativity. 
Economic Evaluation of Anticyclic Citrullinated Peptide Positivity in Rheumatoid Arthritis

\section{TABLE 4 Predicted Cumulative Direct Health Care Cost Differences by Anti-CCP Category ${ }^{a}$}

\begin{tabular}{|c|c|c|c|c|c|c|c|c|}
\hline \multicolumn{9}{|c|}{ Cumulative Years } \\
\hline \multirow[b]{2}{*}{ Number } & 1 & $1-2$ & $1-3$ & $1-4$ & $1-5$ & $1-6$ & $1-7$ & $1-8$ \\
\hline & 2,448 & 1,882 & 1,466 & 1,117 & 809 & 539 & 278 & 83 \\
\hline \multicolumn{9}{|c|}{ Total health care costs, $\$$} \\
\hline Anti-CCP positive & 7,465 & 24,223 & 42,235 & 61,248 & 80,038 & 101,400 & 121,210 & 138,011 \\
\hline Anti-CCP negative & 5,302 & 18,890 & 33,208 & 47,159 & 60,681 & 74,280 & 91,029 & 108,250 \\
\hline Incremental costs ${ }^{\mathrm{b}}$ & 2,163 & 5,333 & 9,027 & 14,089 & 19,357 & 27,119 & 30,180 & 29,760 \\
\hline \multicolumn{9}{|l|}{ Prescription costs, $\$$} \\
\hline Anti-CCP positive & 6,671 & 18,722 & 31,662 & 45,292 & 59,467 & 75,033 & 90,162 & 104,351 \\
\hline Anti-CCP negative & 4,172 & 11,667 & 20,704 & 29,485 & 37,838 & 46,611 & 56,613 & 68,482 \\
\hline Incremental costs ${ }^{\mathrm{b}}$ & 2,499 & 7,054 & 10,957 & 15,806 & 21,628 & 28,422 & 33,549 & 35,870 \\
\hline \multicolumn{9}{|l|}{ Laboratory costs, $\$$} \\
\hline Anti-CCP positive & 398 & 1,288 & 2,173 & 3,070 & 3,920 & 4,739 & 5,588 & 6,329 \\
\hline Anti-CCP negative & 215 & 1,042 & 1,826 & 2,634 & 3,429 & 4,190 & 5,125 & 6,215 \\
\hline Incremental costs ${ }^{\mathrm{b}}$ & 183 & 246 & 348 & 435 & 490 & 550 & 463 & 115 \\
\hline \multicolumn{9}{|c|}{ Rheumatologist visit costs, $\$$} \\
\hline Anti-CCP positive & 298 & 667 & 1,023 & 1,349 & 1,705 & 1,988 & 2,248 & 2,580 \\
\hline Anti-CCP negative & 222 & 480 & 712 & 958 & 1,168 & 1,362 & 1,604 & 1,851 \\
\hline Incremental costs ${ }^{\mathrm{b}}$ & 76 & 187 & 311 & 390 & 537 & 626 & 644 & 729 \\
\hline \multicolumn{9}{|c|}{ Outpatient office visit costs, $\$$} \\
\hline Anti-CCP positive & 338 & 2,010 & 3,777 & 5,546 & 7,361 & 9,402 & 11,126 & 12,683 \\
\hline Anti-CCP negative & 326 & 2,417 & 4,388 & 6,512 & 8,663 & 10,773 & 13,538 & 16,016 \\
\hline Incremental costs ${ }^{\mathrm{b}}$ & 12 & -407 & -611 & -965 & $-1,302$ & $-1,371$ & $-2,412$ & $-3,333$ \\
\hline
\end{tabular}

${ }^{a}$ Cost differences are shown in 2015 U.S. dollars.

${ }^{b}$ Incremental costs $=$ anti-CCP positive - anti-CCP negative

$C C P=$ cyclic citrullinated peptide.

\section{Discussion}

This study showed that anti-CCP positivity was associated with a higher economic burden not only for the short term but also for longer periods of time following a first RA diagnosis. Incremental costs associated with anti-CCP positivity are similar to a previous study for the first 12 months following RA diagnosis. Shafrin et al. (2018) investigated EMRs linked with claims data in 859 RA patients and estimated $\$ 2,698$ in additional RA-associated costs for anti-CCP-positive patients compared with anti-CCP-negative patients. ${ }^{20}$ However, Shafrin et al. did not find differences in all-cause costs, only RA-associated costs. Instead, our study estimated a $\$ 2,163$ overall increase in health care costs; the increase in costs from anti-CCP positivity was consistent throughout the follow-up years, and the differences were even greater in later years.

The economic burden was mainly due to prescription costs. Our study found almost twice as many biologic DMARD users in the anti-CCP-positive group compared with the antiCCP-negative group. These findings are consistent with the previous U.S. study and suggest that patients with anti-CCP positivity may indeed have persistent joint inflammation that leads to earlier initiation of biologic DMARDs. ${ }^{20}$ Moreover, a higher percentage of anti-CCP-positive patients initiated biologic DMARDs within 1 year following their first diagnosis compared with anti-CCP-negative patients. These findings may suggest that anti-CCP-positive patients initiate biologic DMARDs earlier, which is in line with another study that reported that anti-CCP-negative patients experience delays in DMARD initiation. ${ }^{35}$ These findings highlight the importance of cost-effective treatment strategies for anti-CCP-positive patients to further reduce the long-term economic burden. With recent studies suggesting therapies with better treatment response in anti-CCP-positive patients, future studies may address cost-effective treatment options in this population. ${ }^{36,37}$

Anti-CCP positivity was also associated with a higher burden of laboratory testing and more frequent rheumatologist visits, although the additional costs due to laboratory testing and rheumatologist visits were relatively small compared with prescription costs. These may be associated with a higher percentage of biologic DMARD use among anti-CCP-positive patients compared with anti-CCP-negative patients, since patients on biologic DMARDs are often monitored more closely than patients with conventional DMARDs only. ${ }^{38}$ Hospitalizations and ED visits were not increased with anti-CCP positivity even during long-term follow-up; theoretically, early use of biologic DMARDs should reduce erosive RA disease and decrease the need for future hospitalizations and downstream costs. Our inability to identify reduced 
hospitalizations with higher biologic DMARD use may reflect our population of newly diagnosed (incident) RA patients, the low overall use of biologic DMARDs, and the median 3.7 years of follow-up.

Contrary to the study by Shafrin, et al., our study found that anti-CCP-negative patients had higher health care utilization in the 12 months before their RA diagnosis, which was potentially due to a higher comorbidity burden. ${ }^{20}$ In our study, anti-CCPnegative patients had more comorbid conditions, which likely led to the higher number of office visits and hospitalizations during the baseline period.

\section{Limitations}

This study has several limitations. First, this study did not evaluate disease severity using assessment indices such as the Disease Activity Score 28, the Health Assessment Questionnaire, or joint erosion due to limitations of the data. Therefore, we were not able to differentiate whether the higher use of biologic DMARDs in the anti-CCP-positive group was due to disease severity or early initiation of aggressive treatment to prevent joint erosion. Future studies are necessary to disentangle the benefits of early biologic DMARD use among anti-CCP-positive patients on both clinical and economic outcomes.

Second, this study did not include indirect costs in RA patients. Mennini et al. (2017) reported that $45 \%$ of expenditures in RA were due to indirect costs; however, this study was not able to measure indirect costs such as work productivity loss. ${ }^{19}$

Third, RA-specific costs were not calculated, since no reliable algorithm to distinguish RA-specific health care utilization is available. Previous studies defined RA-specific utilization as any encounter with an RA diagnosis code ${ }^{20}$; however, the reliability of this criterion was not validated. Instead, we reported rheumatologist visits or biologic DMARD use, which may be more relevant measures for RA-specific costs.

Fourth, due to the nature of the retrospective observational study design, we could only evaluate the association between anti-CCP positivity and economic outcomes rather than causality. With these limitations, our study findings need to be interpreted with caution.

Fifth, the median length of follow-up was 3.6 years for antiCCP-positive and 3.9 years for anti-CCP-negative patients. Despite using inverse probability weighting for missing values, unobservable factors associated with a higher loss to follow-up in the anti-CCP-positive group could have affected study results.

Sixth, the parallel trend between the anti-CCP-positive and anti-CCP-negative groups was assumed for DID analysis. ${ }^{39}$ This assumption might not hold if the cost outcome trends are very different for anti-CCP-positive and anti-CCP-negative groups at baseline, before anti-CCP testing. We believe that this assumption is reasonable, since anti-CCP testing is a standard test for all newly diagnosed patients with RA, and it is unlikely that other health care utilization or costs are leading anti-CCPpositive test results.
Seventh, this study included patients who received a prescription for DMARD therapy within 12 months from the first diagnosis date due to high positive predictive values of identifying RA. However, this may have excluded patients with more mild disease or those who experienced a delay in initiating treatment.

Finally, although this study suggests a higher economic burden associated with anti-CCP positivity, the majority of the increase was related to prescription costs, which are only an indirect measure of RA disease severity. This could also be an indicator of early aggressive management in anti-CCP-positive patients. Patient-reported outcomes and/or disease progression measures associated with this economic burden may help to further guide treatment decisions.

Despite these limitations, our study has several strengths. We investigated a relatively large group of RA patients with longer follow-up (up to 8 years) than previous studies. To our knowledge, this is the first study to investigate long-term economic outcomes by anti-CCP status at the time of RA diagnosis. In addition, the relatively rich EMR dataset enabled us to investigate patient characteristics and health care utilization patterns in patients with RA. Various statistical approaches were applied to further compare short-term and long-term health care expenditures in the presence of confounding factors and censoring.

\section{Conclusions}

In newly diagnosed RA patients, anti-CCP positivity was associated with a greater economic burden. The increase in economic burden from anti-CCP positivity was consistent across all years of follow-up and was mainly driven by higher prescription costs.

\section{Authors}

JAEJIN AN, BPharm, PhD, and JENNY KANG, PharmD, Department of Pharmacy Practice and Administration, Western University of Health Sciences, Pomona, California. ZOE BIDERCANFIELD, MPH, and T. CRAIG CHEETHAM, PharmD, MS, Department of Research and Evaluation, Kaiser Permanente Southern California, Pasadena. EVO ALEMAO, RPh, PhD, and SEAN E. CONNOLLY, PhD, Health Economics \& Outcomes Research, Bristol-Myers Squibb, Princeton, New Jersey. ANTONY T. LIN, MD, Fontana Medical Center, Southern California Permanente Medical Group, Fontana.

AUTHOR CORRESPONDENCE: Jaejin An, BPharm, PhD, Department of Research E Evaluation, Southern California Medical Group, 100 S. Los Robles, 2nd Fl., Pasadena, CA 91101.

Tel.: 626.564.5906; E-mail: Jaejin.x.an@kp.org. 


\section{DISCLOSURES}

This research and manuscript were funded by Bristol-Myers Squibb (BMS). Alemao and Connolly are employees and shareholders of BMS and participated in the design of the study, interpretation of the data, review/revision of the manuscript, and approval of the final version of the manuscript. An and Cheetham received a grant from BMS for this research. At the time of this study, An was employed by Western University of Health Sciences, and Cheetham was employed by Kaiser Permanente Southern California. BiderCanfield, Kang, and Lin have nothing to disclose.

Some study results were presented as a poster at the American College of Rheumatology Annual Meeting; November 5, 2017; San Diego, CA, and at the International Society for Pharmacoeconomics and Outcomes Research Meeting; May 19, 2018; Baltimore, MD.

\section{REFERENCES}

1. Helmick CG, Felson DT, Lawrence RC, et al. Estimates of the prevalence of arthritis and other rheumatic conditions in the United States. Part I. Arthritis Rheum. 2008;58(1):15-25.

2. Pruijn GJM, Wiik A, van Venrooij WJ. The use of citrullinated peptides and proteins for the diagnosis of rheumatoid arthritis. Arthritis Res Ther. 2010;12(1):203

3. Brooks PM. The burden of musculoskeletal disease-a global perspective. Clin Rheumatol. 2006;25(6):778-81.

4. Birnbaum H, Pike C, Kaufman R, Marynchenko M, Kidolezi Y, Cifaldi M. Societal cost of rheumatoid arthritis patients in the U.S. Curr Med Res Opin. 2010;26(1):77-90

5. Pruijn GJM, Vossenaar ER, Drijfhout JW, van Venrooij WJ, Zendman AJW. Anti-CCP antibody detection facilitates early diagnosis and prognosis of rheumatoid arthritis. Curr Rheum Rev. 2005;1(1):1-7.

6. Niewold TB, Harrison MJ, Paget SA. ACPA antibody testing as a diagnostic and prognostic tool in rheumatoid arthritis. QJM. 2007;100(4):193-201

7. Aletaha D, Neogi T, Silman AJ, et al. 2010 rheumatoid arthritis classification criteria: an American College of Rheumatology/European League Against Rheumatism collaborative initiative. Ann Rheum Dis. 2010;62(9):1580-88.

8. Whiting PF, Smidt N, Sterne JA, et al. Systematic review: accuracy of anticitrullinated peptide antibodies for diagnosing rheumatoid arthritis. Ann Intern Med. 2010;152(7):456-64.

9. Nishimura K, Sugiyama D, Kogata Y, et al. Meta-analysis: diagnostic accuracy of anti-cyclic citrullinated peptide antibody and rheumatoid factor for rheumatoid arthritis. Ann Intern Med. 2007;146(11):797-808

10. Sun J, Zhang Y, Liu L, Liu G. Diagnostic accuracy of combined tests of anti cyclic citrullinated peptide antibody and rheumatoid factor for rheumatoid arthritis: a meta-analysis. Clin Exp Rheumatol. 2014;32(1):11-21.

11. An J, Lin A, Bider Z, et al. AB1006 Anti-citrullinated peptide antibodies and rheumatoid factor testing patterns among patients with rheumatoid arthritis in the U.S. Ann Rheumatic Dis. 2016;75:1245-46 [Abstract].

12. Quinn MA, Gough AK, Green MJ, et al. Anti-CCP antibodies measured at disease onset help identify seronegative rheumatoid arthritis and predict radiological and functional outcome. Rheumatol (Oxford). 2006;45(4):478-80 13. De Rycke L, Peene I, Hoffman IE, et al. Rheumatoid factor and anticitrullinated protein antibodies in rheumatoid arthritis: diagnostic value, associations with radiological progression rate, and extra-articular manifestations. Ann Rheum Dis. 2004;63(12):1587-93.

14. Ronnelid J, Wick MC, Lampa J, et al. Longitudinal analysis of citrullinated protein/peptide antibodies (anti-CP) during 5 year follow up in early rheumatoid arthritis: anti-CP status predicts worse disease activity and greater radiological progression. Ann Rheum Dis. 2005;64(12):1744-49.

15. Humphreys JH, van Nies JA, Chipping J, et al. Rheumatoid factor and anti-citrullinated protein antibody positivity, but not level, are associated with increased mortality in patients with rheumatoid arthritis: results from two large independent cohorts. Arthritis Res Ther. 2014;16(6):483.

16. Mjaavatten MD, van der Heijde D, Uhlig T, et al. The likelihood of persistent arthritis increases with the level of anti-citrullinated peptide antibody and immunoglobulin M rheumatoid factor: a longitudinal study of 376 patients with very early undifferentiated arthritis. Arthritis Res Ther. 2010;12(3):R76

17. Jilani AA, Mackworth-Young CG. The role of citrullinated protein antibodies in predicting erosive disease in rheumatoid arthritis: a systematic literature review and meta-analysis. Int J Rheumatol. 2015;2015:728610.
18. Sakkas LI, Bogdanos DP, Katsiari C, Platsoucas CD. Anti-citrullinated peptides as autoantigens in rheumatoid arthritis-relevance to treatment. Autoimmun Rev. 2014;13(11):1114-20.

19. Mennini FS, Marcellusi A, Gitto L, Iannone F. Economic burden of rheumatoid arthritis in Italy: possible consequences on anti-citrullinated protein antibody-positive patients. Clin Drug Investig. 2017;37(4):375-86.

20. Shafrin J, Tebeka MG, Price K, Patel C, Michaud K. The economic burden of ACPA-positive status among patients with rheumatoid arthritis. J Manag Care Spec Pharm. 2018;24(1):4-11. Available at: https://www.jmcp. org/doi/10.18553/jmcp.2017.17129.

21. Koebnick C, Langer-Gould AM, Gould MK, et al. Sociodemographic characteristics of members of a large, integrated health care system: comparison with U.S. Census Bureau data. Perm J. 2012;16(3):37-41

22. Kim SY, Servi A, Polinski JM, et al. Validation of rheumatoid arthritis diagnoses in health care utilization data. Arthritis Res Ther. 2011;13(1):R32. 23. Centers for Medicare \& Medicaid Services. Physician Fee Schedule look-up tool. Available at: https://www.cms.gov/Medicare/Medicare-Fee-forService-Payment/PFSlookup/index.html. Accessed February 18, 2019. 24. Healthcare Cost and Utilization Project (HCUP). Overview of the National (Nationwide) Inpatient Sample (NIS). Available at: https://www. hcup-us.ahrq.gov/nisoverview.jsp. Accessed February 18, 2019.

25. Truven Health Analytics, IBM Watson Health. IBM Micromedex RED BOOK. Database. Available at: https://truvenhealth.com/Training/Product/ IBM-Micromedex-Clinical-Knowledge/IBM-Micromedex-RED-BOOK. Accessed February 19, 2019.

26. U.S. Department of Labor, Bureau of Labor Statistics. Consumer Price Index. Available at: https://www.bls.gov/cpi/. Accessed February 18, 2019. 27. Ting G, Schneeweiss S, Scranton R, et al. Development of a health care utilisation data-based index for rheumatoid arthritis severity: a preliminary study. Arthritis Res Ther. 2008;10:R95.

28. Stuart EA, Huskamp HA, Duckworth K, et al. Using propensity scores in difference-in-differences models to estimate the effects of a policy change. Health Serv Outcomes Res Methodol. 2014;14(4):166-82.

29. Dimick JB, Ryan AM. Methods for evaluating changes in health care policy: the difference-in-differences approach. JAMA. 2014;312(22):2401-02. 30. Basu A, Polsky D, Manning WG. Estimating treatment effects on healthcare costs under exogeniety: is there a 'magic bullet'? Health Serv Outcomes Res Methodol. 2011;11(1-2):1-26.

31. Lin DY. Linear regression analysis of censored medical costs. Biostatistics. 2000;1(1):35-47.

32. Bang H, Tsiatis AA. Estimating medical costs with censored data. Biometrika. 2000;87(2):329-43

33. Ye X, Henk HJ. An introduction to recently developed methods for analyzing censored cost data. ISPOR Connections: Uniting Science and Practice. 2007;13:11-13.

34. Moore BJ, White S, Washington R, Coenen N, Elixhauser A. Identifying increased risk of readmission and in-hospital mortality using hospital administrative data: the AHRQ Elixhauser Comorbidity Index. Med Care. 2017;55(7):698-705.

35. Pratt AG, Lendrem D, Hargreaves B, Aslam O, Galloway JB, Isaacs JD. Components of treatment delay in rheumatoid arthritis differ according to autoantibody status: validation of a single-centre observation using national audit data. Rheumatol (Oxford). 2016;55(10):1843-48.

36. Seegobin SD, Ma MH, Dahanayake C, et al. ACPA-positive and ACPAnegative rheumatoid arthritis differ in their requirements for combination DMARDs and corticosteroids: secondary analysis of a randomized controlled trial. Arthritis Res Ther. 2014;16(1):R13.

37. Sokolove J, Schiff M, Fleischmann R, et al. Impact of baseline anti-cyclic citrullinated peptide- 2 antibody concentration on efficacy outcomes following treatment with subcutaneous abatacept or adalimumab: 2-year results from the AMPLE trial. Ann Rheum Dis. 2016;75(4):709-14.

38. Cush JJ. Biological drug use: U.S. perspectives on indications and monitoring. Ann Rheum Dis. 2005;64(Suppl 4):iv18-23.

39. Zhou H, Taber C, Arcona S, et al. Difference-in-differences method in comparative effectiveness research: utility with unbalanced groups. Appl Health Econ Health Policy. 2016;14(4):419-29. 
APPENDIX A List of ICD-9-CM and GPI Codes for the Study Cohort

\begin{tabular}{|c|c|c|c|}
\hline $\begin{array}{l}\text { List of Autoimmune Conditions Excluded } \\
\text { from the Study Cohort }\end{array}$ & $\begin{array}{l}\text { ICD-9-CM } \\
\text { Codes }\end{array}$ & $\begin{array}{l}\text { List of Autoimmune Conditions Excluded } \\
\text { from the Study Cohort }\end{array}$ & $\begin{array}{l}\text { ICD-9-CM } \\
\text { Codes }\end{array}$ \\
\hline \multicolumn{2}{|l|}{ Psoriatic and similar disorders } & \multicolumn{2}{|l|}{ Purpura and other hemorrhagic conditions } \\
\hline Psoriasis only & 696.1 & Allergic vasculitis & 287.0 \\
\hline Psoriatic arthritis & 696.0 & Primary thrombocytopenia & 287.3 \\
\hline Polymyalgia rheumatica & $725^{\mathrm{a}}$ & Primary thrombocytopenia, unspecified & 287.30 \\
\hline Multiple sclerosis & $340^{a}$ & Immune thrombocytopenia purpura & 287.31 \\
\hline \multicolumn{2}{|l|}{ Thyroiditis } & $\underline{\text { Sarcoidosis }}$ & $135^{\mathrm{a}}$ \\
\hline Hashimoto thyroiditis & 245.2 & \multicolumn{2}{|l|}{ Diffuse diseases of connective tissue } \\
\hline Giant cell arteritis (temporal arteritis) & 446.5 & Systemic lupus erythematosus & 710.0 \\
\hline \multicolumn{2}{|l|}{ Hemolytic anemia } & Systemic sclerosis & 710.1 \\
\hline Autoimmune hemolytic anemias & 283.0 & Sjögren syndrome & 710.2 \\
\hline Regional enteritis (Crohn's disease) & 555.xx & Dermatomyositis & 710.3 \\
\hline Regional enteritis of small intestine & 555.0 & Polymyositis & 710.4 \\
\hline Regional enteritis of large intestine & 555.1 & Erythema nodosum & 695.2 \\
\hline Regional enteritis of small intestine with & 555.2 & Myasthenia gravis & 695.4 \\
\hline large intestine & & Myasthenia gravis without (acute) exacerbation & 358.0 \\
\hline Regional enteritis of unspecified site & 555.9 & Myasthenia gravis with (acute) exacerbation & 358.1 \\
\hline Ulcerative colitis & $556 . x x$ & Drug Name & GPI Code \\
\hline $\begin{array}{l}\text { Ulcerative (chronic) enterocolitis } \\
\text { Ulcerative (chronic) ileocolitis }\end{array}$ & $\begin{array}{l}556.0 \\
5561\end{array}$ & Abatacept & 6640001000 \\
\hline $\begin{array}{l}\text { Ulcerative (chronic) ileocolitis } \\
\text { Ulcerative (chronic) proctitis }\end{array}$ & $\frac{536.1}{5562}$ & Adalimumab & 6627001500 \\
\hline $\begin{array}{l}\text { Ulcerative (chronic) proctitis } \\
\text { Ulcerative (chronic) proctosigmoiditis }\end{array}$ & $\begin{array}{l}556.2 \\
5563\end{array}$ & Anakinra & 6626001000 \\
\hline $\begin{array}{l}\text { Ulcerative (chronic) proctosigmoiditis } \\
\text { Pseudopolyposis of colon }\end{array}$ & 556.3 & Azathioprine & 9940601000 \\
\hline Pseudopolyposis of colon & 556.4 & Certolizumab pegol & 5250502010 \\
\hline Left-sided ulcerative (chronic) colitis & 556.5 & Cyclosporine & 9940202000 \\
\hline Universal ulcerative (chronic) colitis & 556.6 & Cyclosporine modified & 9940202030 \\
\hline Other ulcerative colitis & 556.8 & Etanercept & 6629003000 \\
\hline Ulcerative colitis unspecified & 556.9 & Golimumab & 6627004000 \\
\hline Inflammatory bowel disease & $558.9^{b}$ & Hydroxychloroquine sulfate & 1300002010 \\
\hline (noninfectious gastroenteritis and colitis) & & Infliximab & 5250504000 \\
\hline Celiac disease & 579.0 & Leflunomide & 6628005000 \\
\hline \multicolumn{2}{|l|}{ Chronic liver disease and cirrhosis } & Methotrexate & 6625005010 \\
\hline Primary biliary cirrhosis & 571.6 & Minocycline hydrochloride & 0400004010 \\
\hline Autoimmune hepatitis & 571.42 & Sulfasalazine & 5250006000 \\
\hline
\end{tabular}


APPENDIX B Median Long-Term Costs per Anti-CCP Test Results ${ }^{\mathrm{a}}$

Type of Encounter

Office visit

Baseline $(\mathrm{N}=2,448)$

1 year $(n=2,448)$

2 years $(n=1,882)$

3 years $(n=1,466)$

4 years $(n=1,117)$

5 years $(n=809)$

6 years $(\mathrm{n}=539)$

7 years $(n=278)$

8 years $(n=83)$

Rheumatologist visit

Baseline $(\mathrm{N}=2,448)$

1 year $(n=2,448)$

2 years $(n=1,882)$

3 years $(n=1,466)$

4 years $(n=1,117)$

5 years $(n=809)$

6 years $(\mathrm{n}=539)$

7 years $(n=278)$

8 years $(n=83)$

Laboratory

Baseline $(\mathrm{N}=2,448)$

1 year $(n=2,448)$

2 years $(n=1,882)$

3 years $(n=1,466)$

4 years $(n=1,117)$

5 years $(n=809)$

6 years $(\mathrm{n}=539)$

7 years $(n=278)$

8 years $(n=83)$

Prescriptions

Baseline $(\mathrm{N}=2,448)$

1 year $(n=2,448)$

2 years $(n=1,882)$

3 years $(n=1,466)$

4 years $(n=1,117)$

5 years $(n=809)$

6 years $(\mathrm{n}=539)$

7 years $(n=278)$

8 years $(n=83)$

Total

Baseline $(\mathrm{N}=2,448)$

1 year $(n=2,448)$

2 years $(n=1,882)$

3 years $(n=1,466)$

4 years $(n=1,117)$

5 years $(n=809)$

6 years $(\mathrm{n}=539)$

7 years $(n=278)$

8 years $(\mathrm{n}=83)$

${ }^{a}$ Costs are shown in 2015 U.S. dollars.

$C C P=$ cyclic citrullinated peptide; $I Q R=$ interquartile range.

Median (IQR) Cost per Patient per Year, Anti-CCP Positive, \$

Median (IQR) Cost per Patient per Year, Anti-CCP Negative, \$

\begin{tabular}{rl|rl}
517.90 & $(247.50-960.70)$ & 647.10 & $(338.0-1,190.40)$ \\
\hline 882.80 & $(497.20-1,388.00)$ & 996.80 & $(594.10-1,566.90)$ \\
$1,158.12$ & $(615.31-2,044.03)$ & $1,494.14$ & $(768.08-2,839.53)$ \\
\hline $1,203.85$ & $(545.87-2,172.66)$ & $1,449.80$ & $(772.21-2,899.98)$ \\
\hline $1,175.05$ & $(538.05-2,209.84)$ & $1,637.33$ & $(620.58-2,846.78)$ \\
\hline $1,249.65$ & $(560.57-2,137.75)$ & $1,487.93$ & $(677.75-2,594.53)$ \\
\hline $1,131.38$ & $(529.09-2,164.75)$ & $1,367.96$ & $(549.77-2,834.31)$ \\
$1,198.84$ & $(438.81-2,471.87)$ & $2,032.65$ & $(758.44-3,660.19)$ \\
$1,381.04$ & $(645.52-2,285.39)$ & $1,619.92$ & $(729.55-2,687.88)$
\end{tabular}

$1,381.04 \quad(645.52-2,285.39)$

$1,619.92 \quad(729.55-2,687.88)$

$0.00 \quad(0.00-120.20)$

$321.00 \quad(168.60-547.80)$

$242.07 \quad(80.69-522.86)$

$238.83(0.00-512.79)$

$183.06 \quad(49.17-445.26)$

$161.38 \quad(0.00-442.17)$

$138.72(0.00-410.75)$

$119.78 \quad(0.00-370.87)$

$119.41 \quad(0.00-390.68)$

$519.00 \quad(324.4-796.30)$

$902.00 \quad(553.5-1,339.70)$

$753.86 \quad(443.31-1,124.30)$

$773.64 \quad(439.70-1,148.00)$

$750.80 \quad(464.48-1,160.88)$

$731.17 \quad(465.83-1,090.01)$

728.93 (441.96-1,046.23)

$666.24 \quad(431.93-1,015.33)$

682.83 (388.90-999.53)

$1,240.90 \quad(350.90-3,385.50)$

$3,755.60 \quad(1,797.60-8,372.60)$

$4,031.16 \quad(1,533.02-10,017.44)$

$4,521.10 \quad(1,822.33-11,680.88)$

$4,658.20 \quad(1,870.50-12,113.94)$

$4,897.47 \quad(1,803.27-13,181.51)$

$5,076.84 \quad(1,876.44-15,606.44)$

$5,020.76 \quad(2,077.40-12,385.97)$

$6,404.45 \quad(3,243.31-12,474.58)$

$2,798.40 \quad(1,333.20-6,223.70)$

$6,199.70 \quad(3,563.20-13,259.70)$

$7,126.22 \quad(3,785.34-16,969.54)$

$7,736.66 \quad(4,226.74-20,516.61)$

$8,370.77 \quad(3,887.34-21,515.97)$

$8,820.14 \quad(4,005.57-21,778.83)$

$9,462.65 \quad(4,148.12-27,539.51)$

$9,389.91 \quad(4,535.64-23,026.08)$

$10,959.97 \quad(5,995.52-18,159.01)$
$80.7 \quad(0.00-137.00)$

$271.20 \quad(129.90-440.30)$

$129.94 \quad(0.00-358.6)$

$119.41 \quad(0.00-322.76)$

$84.94 \quad(0.00-358.24)$

$80.69 \quad(0.00-295.60)$

$49.17 \quad(0.00-200.10)$

$14.55 \quad(0.00-329.35)$

$13.59 \quad(0.00-238.83)$

$614.80 \quad(378.80-922.80)$

$822.10 \quad(456.2-1,239.90)$

$697.65 \quad(394.41-1,122.03)$

$695.62 \quad(386.55-1,025.33)$

$654.89 \quad(416.48-1,043.57)$

$642.82 \quad(381.79-1,060.95)$

$651.77 \quad(344.95-1,056.78)$

$771.50 \quad(405.91-1,122.87)$

$657.46 \quad(336.84-1,175.39)$

$2,268.60 \quad(766.0-5,524.50)$

$4,417.30 \quad(2,072.30-8,268.00)$

$4,268.08 \quad(1,672.16-9,272.66)$

$4,568.88 \quad(2,112.35-10,234.34)$

$4,944.90 \quad(1,971.38-10,185.61)$

$5,455.41 \quad(2,290.35-10,483.17)$

$5,377.84 \quad(2,267.92-10,430.39)$

$5,729.22 \quad(2,189.79-11,441.58)$

$6,551.35 \quad(1,713.07-20,308.92)$

$4,244.90 \quad(2,030.70-9,332.30)$

$7,022.10 \quad(3,884.50-12,994.60)$

$8,028.69 \quad(3,977.41-16,553.91)$

$8,680.23 \quad(4,590.96-16,655.74)$

$9,065.76 \quad(4,738.44-17,265.75)$

$9,224.12 \quad(4,844.92-16,698.40)$

$8,925.49 \quad(4,137.93-18,262.83)$

$10,135.41 \quad(4,567.41-25,372.01)$

$11,710.04 \quad(3,848.51-26,335.88)$ 